\title{
Fuzzy Spreadsheet: Understanding and Exploring Uncertainties in Tabular Calculations
}

\author{
Vaishali Dhanoa, Conny Walchshofer, Andreas Hinterreiter, Eduard Gröller, Marc Streit
}

\begin{abstract}
Spreadsheet-based tools provide a simple yet effective way of calculating values, which makes them the number-one choice for building and formalizing simple models for budget planning and many other applications. A cell in a spreadsheet holds one specific value and gives a discrete, overprecise view of the underlying model. Therefore, spreadsheets are of limited use when investigating the immanent uncertainties of such models and answering what-if questions. Existing extensions typically require a complex modelling process that cannot be smoothly embedded in a tabular layout. In Fuzzy Spreadsheet, a cell can hold and display a distribution of values. This integrated uncertainty handling immediately conveys sensitivity and robustness information. The fuzzification of the cells enables calculations not only with precise values but with distributions, and probabilities. We conservatively added and carefully crafted visuals to maintain the look and feel of traditional spreadsheet while facilitating what-if analyses. Given a user-specified reference cell, Fuzzy Spreadsheet automatically extracts and visualizes contextually relevant information, such as impact, uncertainty, and degree of neighborhood, for the selected and related cells. To evaluate its usability and the perceived mental effort required, we conducted a user study. The results show that our approach outperforms traditional spreadsheets in terms of answer correctness, response time, and perceived mental effort for almost all tasks tested.
\end{abstract}

Index Terms-Uncertainty visualization, tabular data, spreadsheet augmentation.

\section{INTRODUCTION}

$S_{\text {to }}^{\text {p }}$ PREADSHEETS, due to their intuitive and direct approach to computation, are one of the most widely used tools for building and formalizing models in areas as diverse as science, finance, and business [22]. However, the precision implicit in traditional spreadsheet cells [35] often hinders users in performing calculations with uncertain values. Established spreadsheet tools-such as Microsoft Excel [27], Google Sheets [16], and Apache OpenOffice Calc [43] do not take uncertainty into account at all or lack features for exploring how uncertainties propagate and combine throughout calculations.

Integrated handling of variability would be helpful, for instance, to model estimated future values [41] or to plan a budget. To cope with the lack of built-in support for working with uncertain information, users often simplify their problems by calculating alternative scenarios, for instance, covering the worst, best, and average cases. This oversimplification of the problem results in a limited understanding of the complete scenario. To better supports users who want to properly include uncertainty in their calculations, a tool must communicate effectively how individual components influence the final result of the calculations-both in terms of expected value and in terms of uncertainty. To be seamlessly integrated into existing workflows, such a tool should

- Vaishali Dhanoa is with Pro2Future GmbH and Johannes Kepler University Linz. E-mail: vaishali.dhanoa@pro2future.at.

- Conny Walchshofer, Andreas Hinterreiter, and Marc Streit are with Johannes Kepler University Linz. E-mail: \{conny.walchshofer, andreas.hinterreiter, marc.streit\}@jku.at.

- Eduard Gröller is with TU Wien and VRVis Research Center. E-mail: groeller@cg.tuwien.ac.at.

Manuscript received November XX, 2020 retain the familiar spreadsheet interface and enhance it with additional information about uncertainty.

Our primary contribution is the Fuzzy Spreadsheet approach, which augments well-established spreadsheets with compact in-cell visualizations. These visualizations allow users to assess probability distributions and trace computational relationships directly within the spreadsheet cells. Users can analyze how uncertainty propagates through the values and explore the effects of hypothetical changes. As a secondary contribution we present the results of a user study which indicates that Fuzzy Spreadsheet is effective in working with uncertain information in tabular calculations.

\section{Characterizing Uncertainty IN Spread- SHEETS}

To discuss the limitations of traditional spreadsheets and to introduce our terminology, we present an example of a spreadsheet that models the resources required for the maintenance of cars (see Figure 1). We use this spreadsheet as a guiding example throughout the paper. Let us assume that a classic-car enthusiast wants to manage the predicted costs for two of her cars. For the first car, she sums the cost of the checkup and the cost of replacing its expendable parts. For the second car, she plans to upgrade the engine. To reflect this, she enters the cost of buying a new engine and subtracts the selling price of the old one, which results in the total cost for Car 2. To obtain the Grand Total Cost, she combines the summed cost of each car.

To make the underlying network of cells more transparent and to understand which cells influence other cells, we use a computational graph [48]. Figure 2 shows the 


\begin{tabular}{lrrrrr} 
& $\begin{array}{r}\text { Cost (in 1000 } \\
\text { Euros) }\end{array}$ & $\begin{array}{rrrr}\text { Likelihood } \\
\text { Car 1 }\end{array}$ & $\begin{array}{r}\text { Time Spent (in } \\
\text { hours) }\end{array}$ & $\begin{array}{r}\text { Standard } \\
\text { Deviation }\end{array}$ \\
\hline Checkup & 3 & 1 & 5 & 1.5 \\
\hline Spare Part A & 2 & 0.75 & 2 & 1 \\
\hline Spare Part B & 1.5 & 0.5 & 3 & 0.5 \\
\hline Total & 6.5 & 0.38 & 10 & 1.87
\end{tabular}

Car 2

\begin{tabular}{lrrrr} 
New Engine (SP) & 3 & 1 & 3 & 1 \\
Old Engine (CP) & 1 & 0.8 & 1 & 0.5 \\
\hline Total & 2 & 0.80 & 4 & 1.12
\end{tabular}

Grand Total 8.5

0.30

14 2.18

Fig. 1. A simplified spreadsheet for planning the maintenance of two classic cars, containing cost and time required for the maintenance of each car and uncertainty values provided in the adjacent columns.

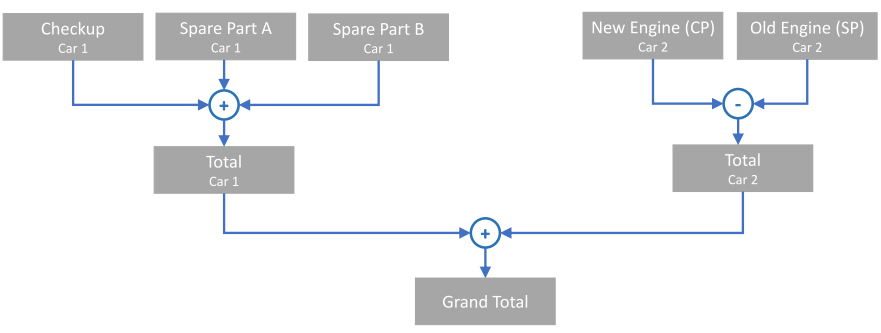

Fig. 2. Computational graph of the example Figure 1 showing the relationships between cells.

computational graph of the example above that models the relationships between cells and highlights the functional expressions that combine them.

Based on experience with Car 1 in the previous years, she knows that each spare part has a different chance of failing within a year. This makes the cost uncertain, as it will only be incurred if the spare part fails. For the second car, her friend shows an interest in buying the engine for the price specified. However, as the deal has not yet been closed, she captures this uncertainty by adding a likelihood of 0.8 in the adjacent column. She computes the likelihood of the total cost by multiplying the individual likelihoods of the input costs. She observes that, according to the spreadsheet (shown in Figure 1), the Total Cost (Car 1) is $6.5 \mathrm{k}$ Euros with a $38 \%$ likelihood. However, since such a calculation covers only one of many possible paths in a probability tree, she receives no indication of the remaining $62 \%$ case(s). In order to gain a complete overview of the actual cost, all the influencing costs must be combined correctly into a distribution. Furthermore, the contribution of each influencing cost on the distribution of the Total Cost (Car 1) can be analyzed if each influencing cost is treated as a distribution rather than a scalar value.

As a traditional spreadsheet lacks this ability to treat a cell as a distribution, she must somehow keep track of the underlying distribution herself. The distribution of an independent cell, such as Spare Part A (Car 1), which is not influenced by other cells, may still be easy to understand.
However, as more of these cells are combined by using formulas to produce cells such as Total Cost (Car 1) and Grand Total, the calculation becomes increasingly complex. The functional expressions in these cells, in this case "sum", no longer perform computations on scalar values, but on distributions. Hence, the Total Cost (Car 1) is then obtained as the sum of individual distributions. In the absence of additional visual cues, it becomes difficult to estimate how the uncertainty propagates from independent cells (Spare Parts A \& B (Car 1)) to the cells which depend on them (Total Cost (Car 1)). Furthermore, the car owner decides to include the time it would take her to inspect and later fix or remove each part to avoid paying a professional car mechanic. She enters the time required for each part. However, as each part has a different chance of failing, the time spent on each part varies accordingly. As when calculating cost, temporal variability must be considered and can be modeled as a distribution by specifying a standard deviation for each part in an adjacent column. The Total Time spent is then computed by adding the influencing distributions. Now, she has to monitor not only the uncertainty in cost, but also in time. Without a proper way to model them in the spreadsheet, it becomes difficult to keep track of the distributions and how they propagate throughout the spreadsheet. With the Fuzzy Spreadsheet approach, users are able to achieve these tasks.

In some cases, it may be relevant to analyze the impact a cell has on another, for instance, to identify highly influential cells such as Total Cost (Car 1) and its impact on the Grand Total. Conceptually, a cell's impact can be defined as how much its value contributes to the value of another cell. In some cases, it is sufficient to simply look at the values of the cells to identify those with the highest impact. However, determining the exact impact a cell has on another requires calculation. We compute this impact as a percentage of how much the value $x_{i}$ of cell $i$ contributes to the value $x_{j}$ of cell $j$. If $i$ is a first-degree influencing neighbor of $j$ (i.e., $i \in \mathscr{N}_{1}(j)$, then we define its impact as:

$$
\mathscr{I}(i \rightarrow j)=\frac{\sigma \cdot x_{i}}{\sum_{k \in \mathscr{N}_{1}(j)}\left|x_{k}\right|} .
$$

Here, $\sigma=+1$ if $i$ contributes to $j$ via a summation, and $\sigma=-1$ if $i$ contributes to $j$ via a difference. The total relative sum of the impact percentages of each contributing cell $c_{i}$ must be $100 \%$. This direct computation involving simple addition or subtraction is straightforward but longer calculations involving intermediate results, such as a difference of sums, can make assessing the impact challenging. We categorize the nature of this impact as positive or negative based on the sign and relationship of the cell $j$ with $i$. In a spreadsheet, an additional column may be created to compute the impact that value $x_{i}$ in cell $i$ would have on value $x_{j}$ of cell $j$. For a cell $i$, as its impact value influences $j, j$ must be re-evaluated every time $i$ changes.

Finally, it can often be helpful to explore the results of hypothetical changes to the spreadsheet to answer questions such as "What would happen to the Grand Total Cost if I were to change the chance of failure of Spare Part A of Car 1 from 0.75 to 0.5 ?" The model must be able to incorporate the new uncertainty for Spare Part A of Car 1 and propagate the change through the computational graph. This analytical concept of what-if analysis often involves 
TABLE 1

Terminology used for describing uncertainty and other relevant concepts.

\begin{tabular}{ll}
\hline Term & Definition \\
\hline Standard Deviation & $\begin{array}{l}\text { Uncertainty of cells modeled via a normal distribution } N\left(\mu, \sigma^{2}\right) \text { with user-provided values for mean } \mu \\
\text { and standard deviation } \sigma\end{array}$ \\
\hline Likelihood & Probability $p=P(X=A)$ that a variable $X$ modeled via a Bernoulli distribution assumes the value $A$ \\
\hline Distribution & General description of uncertain variables, assigning a probability density to each possible value \\
\hline Relationship & $\begin{array}{l}\text { Relationship between a cell and other cells based on the direction of influence (input/output) and the } \\
\text { degree of neighborhood }\end{array}$ \\
\hline Degree of Neighborhood (DON) & Distance between two cells in the computational graph \\
\hline Impact & How much the value of a cell contributes to the value of another cell, expressed as percentage \\
\hline
\end{tabular}

changing the certainty of values to create and analyze different scenarios. Such an analysis requires considerable mental effort if intermediate changes are not tracked and no visual clues are provided. With Fuzzy Spreadsheet, we aim to support users in maintaining their mental models during calculations involving uncertainty. Brief descriptions of the key terms introduced in this section are given in Table 1.

\section{Related Work}

Uncertainty is an inherent part of data collection, processing, and sensemaking. When dealing with uncertainty, visualization is an indispensable tool for communicating effectively both the data itself and the underlying uncertainty. Exploring and understanding variabilities may have a considerable impact on the outcome of decisionmaking [2], [21]. Research into how uncertain values can be visualized effectively has been ongoing for centuries [3], [33]. Various uncertainty visualization techniques have been applied in a diverse range of fields, such as medicine [25], geoscience [39], business intelligence [45], and fishery [36]. As technology develops rapidly, tools are proliferating, but current applications focus on showing complex information and do not make use of the familiar spreadsheet layout to present different scenarios-such as worst, best, and expected case-in a way that is easy to understand and visualize [34], [37]. With our work, we aim to deepen the understanding of uncertain values and their use in tabular calculations.

\subsection{Uncertainty Visualization}

Uncertainty can be visualized for zero-, one-, or higherdimensional data [37]. Sanyal et al. $|38|$ introduced a framework for data dimensionality (0D, 1D, 2D, 3D), visualization approaches (scalar, vector, tensor), and uncertainty visualization techniques (e.g., blurring, transparency, noise). Typical techniques for showing zero-dimensional data (after decoupling of the temporal dimension) include changes in glyph size to indicate a variation in the data points [4]. As for one-dimensional data, line blurring and transparency changes are common techniques to indicate the level of certainty [38]. Further, probability density functions [7], [10] are used to represent uncertainty by, for instance, line and bar charts [40], [44], dotplots [11], violin/boxplots [18], or heatmaps |19|.

\subsection{Spreadsheet-based Tools}

The suitability of a particular type of uncertainty encoding depends on the application. For the purpose of this work, we limit the discussion of related work to spreadsheetbased tools that address the encoding and propagation of uncertainty information.

Streit et al. [41] proposed a technique for augmenting a spreadsheet with uncertain information. To introduce uncertainty in the calculations, users can specify an interval or range in a cell. The underlying theoretical concept is known as interval arithmetic. The uncertainty information is then propagated to other cells that use these ranges as inputs. To convey to the user that-as an effect of the propagationthese cells now contain intervals, affected cells are highlighted by shading. Finally, these intervals are plotted as a chart that represents the uncertainty.

The FuziCalc tool [8], aimed at modeling under uncertainty, followed a similar approach. FuziCalc allowed the user to enter fuzzy inputs in the form of shapes, such as triangles representing best, worst, and expected cases. It then generated a fuzzy output from fuzzy input cells. This way, it reduced the mental effort for users by not exposing them to the complex underlying computations. Since FuziCalc was developed as an independent spreadsheet tool, it missed fundamental features of conventional generalpurpose spreadsheet tools. In most cases, it was used in addition to conventional spreadsheet tools and not as a replacement, which resulted in a cumbersome workflow. Since this commercial software is no longer available, it could not be tested.

More recent tools for uncertainty visualization in spreadsheets are Palisade @Risk [32] and Oracle Crystal Ball [30]. These tools use Monte Carlo simulation to compute possible outcomes of uncertain events [12]. Both applications are commercially available as extensions for Excel, and both allow users to explore different scenarios and provide results in the familiar Excel charts. They share the concept that the user can input a distribution for the cells by using either a formula or a graphical user interface. The user can choose distributions for cells which are not influenced by other cells and compute the model for a reference cell (which is usually a cell that is influenced by input cells). The cells are then colored to indicate that they no longer contain exact but fuzzy values based on probability distributions. More 
information on a distribution appears upon mouse-over action and click-based interaction in the cases of @RISK and Oracle Crystal Ball, respectively. The user can interact with the distribution to analyze the probability of finding a value in a particular range. However, these tools lack an overview of the underlying uncertainty across the spreadsheet, and require a complex modeling process of the distributions for each cell. Furthermore, for users who are not familiar with probability distributions, it is challenging to define and interpret them [15].

A tool that avoids the complexity of the modeling process is Guesstimate [17]. It was developed as an independent web-based spreadsheet tool that allows the user to easily input a fuzzy number as a range, an interval, or a distribution, and immediately provides an output based on the relationship. Relationships between the cells are shown as links connecting the related cells. Similar to Palisade @Risk and Oracle Crystal Ball, it uses Monte Carlo simulation to compute the propagation of uncertainty. In the course of our investigation, we contacted one of the main contributors of Guesstimate. He informed us that users generally prefer staying close to traditional spreadsheet-based approaches and primarily rely on normal distributions due to a lack of knowledge about applying other distributions, even though further ones are supported. This confirmed our findings in the context of the FuziCalc software that users are less likely to model their use cases in an unfamiliar environment. We found that existing tools for uncertainty exploration with what-if analyses are either too complex for a novice user or fail to provide the wide range of features provided by general-purpose spreadsheet tools. This inspired us to develop an approach that (1) retains the layout of a traditional spreadsheet and (2) is augmented with compact visualizations to communicate the uncertainty.

\section{Task Characterization}

We collected the most prevalent user tasks that are supported by various related tools (see Section 3 for a survey) and put these tasks into the context of existing analysis task frameworks [1], [6]. For the purpose of discussing Fuzzy Spreadsheet, we categorize user tasks in two different ways.

First, we assign the tasks to one of two types of phases of the typical spreadsheet workflow: Authoring and Analysis. In the Authoring phase, users set up content and structure of the spreadsheets. In the Analysis phase, users seek to understand the results of calculations and draw conclusions for their applications. Complex problems may require users to go through multiple iterations of Authoring and Analysis phases.

Second, we label user tasks by the requirements they impose on Fuzzy Spreadsheet. In order to facilitate decisionmaking in the face of uncertainty, Fuzzy Spreadsheet must extend traditional spreadsheets in two ways. To reflect on the necessary extensions, we define the following three task requirements:

田 Basic spreadsheet functionality is required for all tasks that can be readily performed in a typical spreadsheet tool without any extensions, such as inputting numbers and referencing cells in formulas.
아 Computational graph parsing is required for all tasks that involve extracting relationship information from the computational graph underlying the spreadsheet.

Fuzzification of the spreadsheet is required whenever tasks cause a transition from exact numbers to probability distributions.

Not all user tasks need to be assignable to exactly one of these requirements. Certain tasks involve basic spreadsheet functionality, but may lead to fuzzification or may pave the way to improved analysis of the computational graph. Other tasks may be equivalent to those performed in nonaugmented spreadsheets, but may acquire a new meaning in the context of uncertainty. Table 2 summarizes the user tasks we identified for spreadsheets with uncertainty propagation and lists their requirements and to which phase we assigned them. The following sections describe the user tasks in more detail.

\subsection{Authoring Tasks}

In the authoring phase, users set up the initial content and structure of the spreadsheets. We extracted four tasks that are essential in this phase. First, users Tabulate Numbers 国. Users choose a layout according to which they input values into cells. In traditional spreadsheet tools, all values are treated as exact numbers. However, in Fuzzy Spreadsheet, cells which are computed as the average of other cells are considered to be uncertain cells. By extension, the cells which are influenced by these average cells are also treated as uncertain cells. Each value is a potential node in the spreadsheet's underlying computational graph. Second, users Set Up Relations Es by inputting formulas, which introduce links between nodes in the computational graph. Third, users Specify Uncertainties E... In this essential step for the fuzzification of spreadsheets, users specify which cells should no longer be treated as exact, but fuzzy values. To the users, this task can be equivalent to a Tabulate Numbers task, with the important conceptual difference that the input numbers are given a special meaning in the subsequent analysis phase. Fourth, users might want to Introduce Alternatives $E$ to treat numbers as potential results of probabilistic binary processes. As explained in Section 2. we borrow the term likelihood from statistics to refer to the chance of such a process having a particular outcome. Again, the act of inputting the likelihood can essentially be a Tabulate Numbers task with the system assigning a special meaning to the numbers. Since Fuzzy Spreadsheet is designed to build upon these authoring tasks, it does not provide any special functionality for supporting them. These tasks must be performed in advance in a spreadsheet program, as they are essential for the analysis phase.

\subsection{Analysis Tasks}

While the authoring tasks are important for setting up content and structure of the spreadsheet, Fuzzy Spreadsheet focuses mainly on enabling new tasks in the analysis phase. The analysis tasks are extracted from the rationale-based tasks identified by Amar et al. [1] and extended to incorporate tasks that played a role in the tools we surveyed. Additionally, we also cover the four search tasks (Lookup, 
TABLE 2

User tasks for Fuzzy Spreadsheet. Tasks are divided into Authoring and Analysis phases, and labeled depending on the requirements.

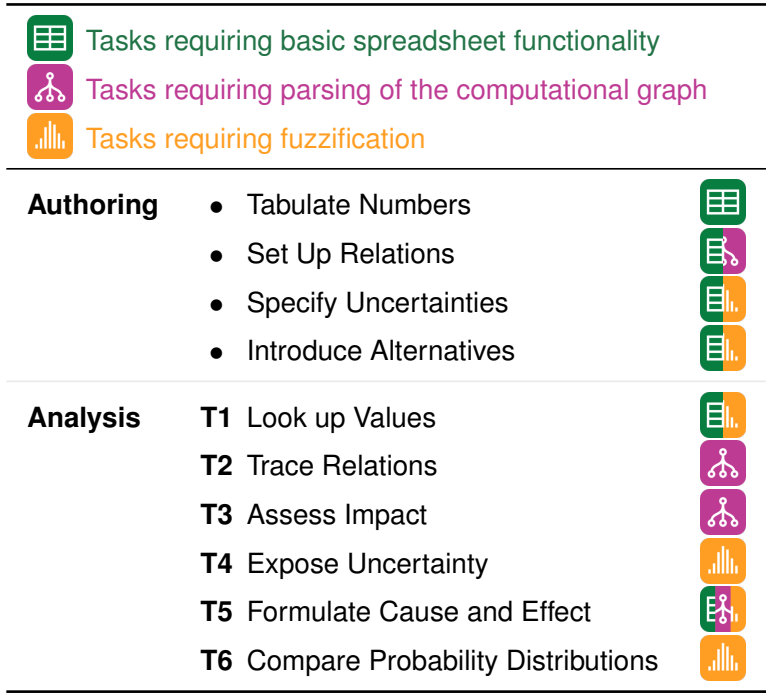

Locate, Browse, Explore) and two query tasks (Identify and Compare) from the Multi-Level Task Typology framework of Brehmer and Munzner [6]. Our discussion of the visual encodings used in Fuzzy Spreadsheet and of the user study results are based on the following analysis tasks:

\section{T1 Look up Values E.}

The most basic analysis task when working with spreadsheets is to look up values in cells. In the same way that input tasks can acquire a special meaning if they introduce uncertainty to the spreadsheet, so do look-up tasks that retrieve these special values.

\section{T2 Trace Relations of}

Tracing relationships between cells in a spreadsheet is important for understanding the "flow" of computations. As discussed in Section 2, this flow is described by the spreadsheet's underlying computational graph. In classic spreadsheet tools, the computational graph is, for the most part, hidden from the user. Given a selected cell, only the immediate inputs used in formulas in that cell can be readily retrieved. This retrieval is only one special case of the more general analysis task Trace Relations, which is a bidirectional task: in addition to finding direct neighbors that influence a given cell, it also includes finding cells that are influenced by other cells. Neither influencing nor influenced by cells are limited to direct neighbors in the computational graph, but can be extended to a higher degree of neighborhood (DoN). If no special interface is provided for determining higherdegree neighbors, they can be found by detecting firstdegree neighbors in a nested and possibly branched manner.

\section{T3 Assess Impact of}

Having located cells that influence another cell, users often need to gain a better understanding of the individual contributions of each of these influencing cells. In Section 2, we introduced the impact as a direct mea- sure of how strongly the value of one cell contributes to the result in another cell. In simple cases, such as the addition of multiple values, assessing the impact can be as straightforward as looking up several values and comparing them. If more elaborate formulas are used, and/or in the case of tracing longer paths in the computational graph, assessing impacts is challenging in standard spreadsheet tools.

\section{T4 Expose Uncertainty}

In the authoring phase, users Specify Uncertainties and Introduce Alternatives. As a result, computations in the spreadsheet can no longer be based on exact numbers but require probability distributions. Users must be able to asses these distributions, preferably directly in the spreadsheet cells without auxiliary calculations. Depending on the level of detail with which they want to analyze these probability distributions, users must perform a number of low-level visualization tasks, such as assessing shapes, finding extrema, and estimating areas under the curve.

\section{T5 Formulate Cause and Effect E:}

While adding uncertainty to spreadsheets allows users to make more informed decisions, it also makes interpreting the relationships between cells in a spreadsheet more challenging. As described in Section 2, a what-if analysis is a powerful approach that allows users to safely explore the results of hypothetical changes in an attempt to better understand which values are important. To enable this, users must be able to change the spreadsheet reversibly. When users end their exploratory analysis, they can decide to either keep the new results or revert back to the initial values. Hypothetical changes typically include changing the mean values, uncertainties, and/or likelihood values of cells while keeping the topology of the computational graph unaltered. Given the density of data in typical spreadsheets, making reversible changes might not be enough for an effective what-if analysis. Users must be able to see how a change in one cell propagates through the computational graph and how each change leads to additional, implicit changes in other cells. Explicitly addressing this subtask by visualizing changes prevents users from having to Trace Relations (T2) repeatedly in the course of a what-if analysis.

\section{T6 Compare Probability Distributions}

In later stages of the analysis, most of the intermediate and final results of calculations in the spreadsheet are modeled by probability distributions. This means that comparing two or more cells requires comparing two or more probability distributions. We refer to this as between-cell comparison. Additionally, hypothetical changes in a cell during a what-if analysis may entail comparing old and new results within a single cell. We refer to this as within-cell comparison.

Fuzzy Spreadsheet addresses most of these analysis tasks by introducing compact visualizations directly in the spreadsheet cells, with additional detailed information in a separate side panel. The between-cell comparison is supported by in-cell encodings. Fuzzy Spreadsheet allows users to compare probability distributions of two or more cells by 
means of individual encodings presented within the cells. Detailed information about each cell, such as the probability distribution with computed mean and standard deviation, can be viewed in the side panel upon selecting a cell. During a what-if analysis, the encodings of the old and the new probability distributions are stacked within a cell, which facilitates a within-cell comparison. The within-cell changes can also be viewed in the side panel.

\section{Fuzzy Spreadsheet Technique}

We designed Fuzzy Spreadsheet as an extension of traditional spreadsheet programs, which enables users to incorporate our solution seamlessly into their familiar workflow. Fuzzy Spreadsheet comprises three parts: (1) functionalities to calculate how the uncertainty information propagates through the spreadsheet; (2) carefully chosen compact visualizations that are embedded directly in the spreadsheet cells; and (3) a side panel with the user interface and additional information. Figure 3 shows an example spreadsheet with some of the Fuzzy Spreadsheet encodings (the traditional spreadsheet version is shown in Figure 11. We first discuss the pre-processing needed for calculating the propagation of uncertainty information, followed by how users control the in-cell visualizations from the side panel, and then describe the design of the visual components in more detail.

\subsection{Computational Graph Parsing and Uncertainty Cal- culations}

Before relational and uncertainty information can be encoded and interacted with, the relevant content must be extracted from the spreadsheet. To this end, Fuzzy Spreadsheet parses the spreadsheet's underlying computational graph. In order to perform an analysis task (see Section 4.2), a user needs to choose a reference cell-typically a cell of interest whose value depends on other cells. The Fuzzy Spreadsheet extension stores the address of this cell and uses it as a reference for all future computations. This gives the user the possibility of selecting another cell and viewing its details with respect to the reference cell. Fuzzy Spreadsheet allows the user to focus on two cells at any given time: a reference cell and a selected cell.

For the analysis tasks, the extension performs the computations required for obtaining impact values and probability distributions, as explained in Section 2 . Based on the headers of the adjacent columns of each uncertain cell, the extension parses the values of standard deviation and likelihood. If an uncertain cell is composed of other uncertain cells, their parsed values are ignored in the calculation of their distributions. The tool performs Monte Carlo simulation to obtain the distributions. Due to computational limitations, we discretize each distribution to 100 samples. During a what-if analysis, after a cell value is changed, an automatic parsing of the computational graph is performed to derive new results for impact, likelihood, and distribution. If the newly obtained results are different from those obtained in the initial parsing progress, the cell encoding for probability distribution and the side-panel visualization for all selected options is updated.

\subsection{Side Panel}

The side panel, as shown in Figure 3(B), provides the control interface for our extension. To start working with Fuzzy Spreadsheet, users need to trigger the parsing and creation of the computational graph by clicking on the Initialize button. Users must then select a cell in the spreadsheet and mark it as the reference cell. The reference cell is automatically highlighted with an orange border in the spreadsheet (see Figure 3(D)), and its address is shown in the same color in the upper-right corner of the side panel.

Next, users select which kind of cells they are interested in: cells influencing the reference cell or cells that are influenced by the reference cell. Subsequently, they choose the degree of neighborhood (DoN) up to which they want to explore related cells. A DoN of zero refers to the reference cell itself. A DoN of one corresponds to the direct neighbors (connected by links in the computational graph) of the reference cell, excluding the reference cell itself. A DoN of two corresponds to the direct neighbors of the direct neighbors, excluding the cells with a DoN of one. Up to this point, no visual changes have appeared in the spreadsheet. To indicate that a DoN of zero limits the selection to the reference cell itself, the zero option is colored in the same orange hue that is used for the borders of reference cell. This neighborhood-selection dialog simultaneously serves as a legend for the relationship encoding (see Section 5.4). We refer to this dialog/legend hybrid as active legend. In our Excel extension prototype (see Section 6), we use a dark green shade for all information related to the selected cell to keep the design consistent with Excel's default color setting for the border of the selected cell (see Figure $3(\mathrm{C})$ ).

Based on this information, users can display visualizations of impact, likelihood, and distribution for the selected and related cells on demand. At any point, they can change the DoN, which controls the overall amount of information displayed. Furthermore, users can start a what-if analysis by activating a button at the bottom of the side panel. We explain for each visualization separately how this analysis mode affects the encoding.

\subsection{Compact Visual Encoding}

Given the small cell size in traditional spreadsheets, we had to evaluate encodings that work effectively in this constrained space. Sparklines and glyph-based encodings [4] are one option. Nobre et al. |29| introduced an aggregated layout in tabular cells that could be adapted to show fuzzy values within a cell. Box plots, heatmaps, sparklines, histograms, and dotplots used in Taggle by Furmanova et al. [13] are also possible choices for in-cell encodings. All these visualizations inspired our final encodings. The cell encodings are designed to give overview information about the reference cell and its related cells-serving as an initial guidance in identifying critical cells. We justify our final choice of encoding for each of the suggested visualizations in individual sections below.

\subsection{Relationship Encoding}

To enable Trace Relations tasks (T2), each cell of interest is equipped with a relationship marker. The shape of this 

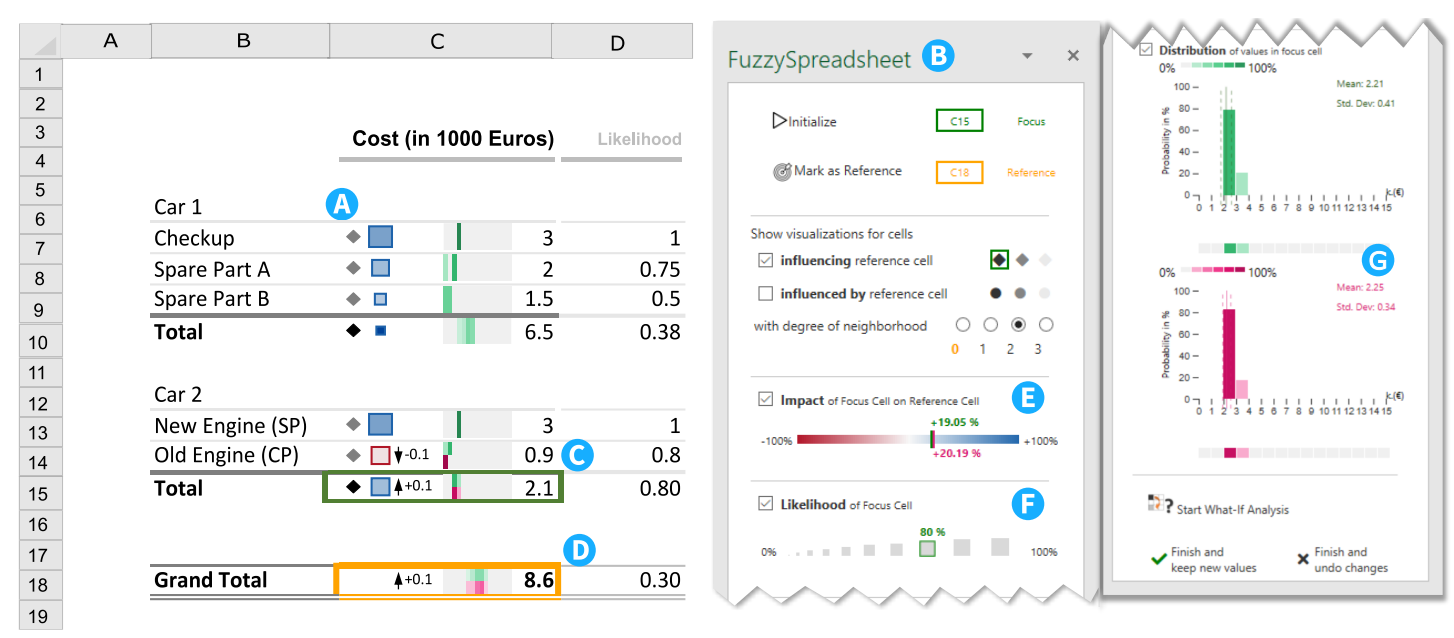

Fig. 3. Fuzzy Spreadsheet applied to the car-maintenance subset from the example in Figure 1 The in-cell visualizations indicate (A) impact $\square \square \square \square \square$, likelihood . $\square \square \square \square$, relationship $\bullet \bullet$, and probability distributions control the visualizations. Users select a cell (C) and explore its influence on a reference cell (D). Additional information is displayed in the active legends $(E)$ for impact and $(F)$ for likelihood with dark green markers indicating original values and dark pink markers indicating new values obtained after the what-if analysis, where the user reduces the value of Old Engine by 0.1 . The detailed distribution charts (G) for the original and the new values of $(\mathrm{C})$ are also displayed, and the probability ranges, can be viewed by hovering over them.

marker encodes the direction of influence with respect to the reference cell. A diamond marker represents a cell that influences the reference cell, and a disk represents a cell that is influenced by the reference cell. We chose these shapes to avoid cross-talk with the impact and likelihood encodings. The side panel also emphasizes this information by outlining the marker in dark green if a related cell is selected.

Additionally, the DoN is encoded in the brightness of the marker. Cells with a DoN of one (i.e., immediate neighbors in the computational graph) are represented by black markers, with lighter shades of gray for for cells with a DoN of two and three.

In the design process, we considered two alternatives to the categorical relationship markers. First, we considered drawing lines that connect related cells, which would have represented the underlying node-link structure of the computational graph. In Excel, users already have the option to turn on this kind of encoding as an overlay. However, we found that this encoding caused visual clutter and was prone to drastic changes depending on the layout (i.e., positioning) of the cells chosen by the user in the Authoring phase. Second, we considered small arrow-like glyphs or stubs [23] pointing towards or away from the reference cell. While this encoding was easy to understand in simple cases, we discarded it because identical glyphs would have acquired different meanings depending on their locations in the spreadsheet. The current encoding has the advantage that it is fully independent of the spreadsheet layout chosen by the user in the Authoring phase, and the markers make it easy to quickly spot the cells of interest.

\subsection{Impact Encoding}

Impact markers enable users to Assess Impact (T3). We add boxes of fixed size on the left side of a cell and color them according to how strongly the value in the cell influences the reference cell (see Figure 3(A)). We use a diverging color scale from dark red (strong negative impact) to white (no impact) and dark blue (strong positive impact), to help users to quickly identify the sign of a cell's contribution to the reference cell. Furthermore, the dark colors at each end of the scale make high-impact cells more salient, while cells with an (almost) white impact marker can be safely disregarded by users while they are gaining an overview.

The side panel also serves as an active legend for the impact. The active legend consists of a red/blue color scale (see Figure 3(E)). Using a dark green marker, the legend shows - for a selected cell-either its impact on the reference cell or the impact of the reference cell on it. Using the same dark green which is used as the border color of the selected cell, the legend also shows the value of the impact in percent. The position of this marker changes dynamically when the selected cell is changed. To support Formulate Cause and Effect tasks (T5 during a what-if analysis, a second, dark pink marker is shown in the active legend if any of the hypothetical changes affect its value, as indicated in the legend in Figure 3(E).

\subsection{Likelihood Encoding}

- $\square \square \square \square$

We encode the likelihood associated with a cell by a square marker shown on the left side of the cell. The likelihood value determines the area of this marker. If only the likelihood visualization but not the impact visualization is switched on, we show gray likelihood markers in corresponding sizes. If both likelihood and impact visualizations are switched on, we encode the likelihood in the size of the impact marker and use the color from the impact encoding.

The side panel also functions as an active legend for the likelihood. The active legend for the likelihood consists of grey squares placed in ascending order of their length. For a selected cell, we show its likelihood value (in percent) in the legend and enframe its corresponding square with a dark green box, as seen in Figure 3(F). As for the impact encoding, a second, dark pink marker in the active legend indicates changed likelihood values during what-if analyses. 


\subsection{Probability Distribution Encoding}

Users can switch on the probability visualization in the side panel to show small, in-cell distribution heatmaps attached to the cells of interest (see Figure 3). The heatmaps serve as a first visual aid for Expose Uncertainty tasks (T4). Each heatmap is based on a binned histogram of samples drawn from a continuous probability distribution $p(x)$. The value $x$ increases from left to right, and the heatmap is divided into a fixed number of intervals $I$ (i.e., the bins). The range of the heatmap is based on the minimum and maximum cell value present in the spreadsheet, and is the same for all cells to enable an unbiased comparison. Due to the limited space, the range cannot be shown numerically or quantitatively within a cell. However, as the vertical space in the side panel is not limited, we indicate the range of the heatmap on a shared $x$-axis with a bar chart for the detailed distribution plot, as shown in Figure 3.G). The horizontal orientation of the distribution heatmaps facilitates comparison between values of cells in the same column and uses the space provided by typically sized spreadsheet cells optimally. The color scale for the heatmaps is based on the selected cell color (by default dark green in Excel), and each interval is colored according to the binned probability $P(x)$, where $x \in I$. Thus, higher probabilities are represented by darker shades of the base color. Zero is represented by a neutral grey, which lets users distinguish easily between improbable (light-colored) and impossible (gray) values.

Similarly, we color each bar in the detailed distribution plot in the side panel in the same shade of the base color as in the heatmap, and align a copy of the heatmap with the bottom of the bars. This allows users to easily match the heatmap encoding in the spreadsheet with the barchart encoding in the detailed view. Mouse-over interactions let users assess exact probability values for each interval. Additionally, we add the mean and standard deviation of the shown samples as numbers and indicate these values by vertical lines in the bar chart. The distribution bar chart in the side panel enables Expose Uncertainty tasks (T4) for which the heatmap encoding cannot provide sufficient detail.

In the course of a what-if analysis, changes made by the users often affect the probability distributions of cells. To let users assess these changes at a glance, we replace each heatmap with a stacked heatmap (see Figure 3 (C) or thumbnail at the beginning of this section). The stacked heatmaps allow users to Compare Probability Distributions (T6) and Formulate Cause and Effect (T5) directly in the spreadsheet. We show the original distribution heatmap in green in the top half of the cell, and the updated distribution in pink in the bottom half. Both heatmaps have the same value range as the original heatmap. Similarly, we change the detailed view in the side panel from a single bar chart to two bar charts, representing the original and the updated distributions (see Figure $3(\mathrm{G})$ ). The base hue used in the updated distribution heatmap and bar chart is the same shade of pink that encodes the updated impact and likelihood values in the active legends.

In the design process, we also considered using sparklines to visualize the probability distributions. We chose the heatmap encoding instead for two reasons. First, the vertical space in the cells is constrained. As heatmaps encode the values in the color channel, they require little vertical space compared to bar charts or sparklines, which rely on the vertical position for encoding the values. Additionally, during a what-if analysis two distributions must often be shown for a cell at the same time. We found that a stacked heatmap encoding uses the space more effectively and with less visual clutter that overlaid sparklines. Second, heatmaps are particularly suited to comparing distributions (T6) across vertically neighboring cells [14].

\subsection{Change Indicators}

$\mathbf{4}+21.8 \quad \downarrow-18$

Not all Formulate Cause and Effect tasks (T5) necessarily involve tracking changes in impact, likelihood, and/or probability distributions. In many cases, it might be sufficient to simply see how the value of a cell is affected by changes made elsewhere. During a what-if analysis, we display small arrows to indicate increases (upward arrow) or decreases (downward arrow) as a result of the changes (see Figure 3. C) or thumbnail above). Additionally, the value difference is specified next to the arrow.

\section{IMPLEMENTATION}

For the implementation, we investigated the most commonly used spreadsheet tools and evaluated them according to their official API extension support and feature list. Microsoft Excel emerged as our tool of choice because it provides the Office JavaScript API. We used the Host Specific Office API model for Excel [28], which allows our extension to be loaded as a so-called add-in in both the desktop and the web version. The add-in currently supports Office 365 and the Microsoft Edge Web Browser. We implemented the prototype in TypeScript, HTML, and CSS. The source code is available at https://github.com/ jku-vds-lab/fuzzy-spreadsheet A demo version can be accessed at https://jku-vds-lab.at/fuzzy-spreadsheet/ Our prototype uses external libraries, the most notable ones being discrete-sampling [26] for sampling discrete probability distributions, jstat [46] for performing advanced statistical operations, maths.js [9] for flexible expression parsing, and D3.js [5] for creating customized visualizations.

\section{USAGE SCENARIO}

To demonstrate the applicability of Fuzzy Spreadsheet, let us assume that conference organizers have been assigned the task of planning the budget for an annual conference. Using a simple model, the organizers predict the revenues, expenses, and the balance for the next year as the average of the previous six conferences (see Figure 4). As planning is prone to uncertainty, decisions on the variation of all uncertain values must be made and conveyed in the spreadsheet. Since the spreadsheet itself does not offer much support for dealing with uncertain data, the organizers decide to use the Fuzzy Spreadsheet extension. Due to its easy modeling requirements, they simply create two additional columns to enter the uncertainty parameters, namely standard deviation and likelihood. For each uncertain value, they specify the value of standard deviation where required. The organizers expect a likelihood value of one ( $100 \%$ likelihood) for all uncertain values except for Venue and Catering. These 

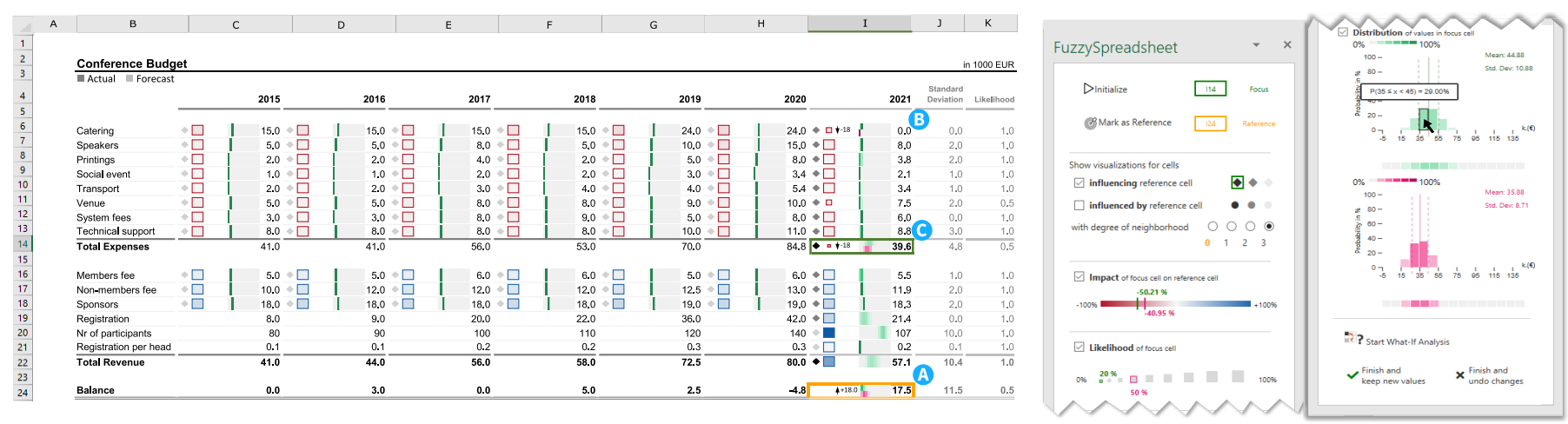

Fig. 4. Conference planning usage scenario with Fuzzy Spreadsheet encodings. The user selects as a reference cell (A) and controls the visualization using the side panel. In the what-if analysis mode, they reduce the value of Catering 2021 to zero (B) and analyze its effect on the focus cell $(\mathrm{C})$. More detailed information for the focus cell is shown in the side panel (right).

two costs will only occur if the conference takes place on site. Since unexpected worldwide events have recently led to travel restrictions, they estimate the likelihood for an onsite conference-and therefore for the Venue and Catering costs-as $50 \%$.

To visually analyze the effect of uncertainty on the balance and the intermediate calculations, they load the Fuzzy Spreadsheet extension. Conference organizers tend to aim for a slightly positive balance, using any surplus for the next conference or better catering. The organizers start the analysis by clicking on the Initialize button, which automatically parses the computational graph of the spreadsheet, and mark the summed balance as the reference cell (see Figure $4(\mathrm{D}))$.

The organizers can now decide which visual analysis support they want to activate in the side panel: relationship, neighborhood, impact, likelihood, and/or distribution. To determine which cells influence the uncertainty of the balance (T4), they first display the influencing cells (T2) and change the DoN to two. The glyphs that appear within the cells indicate the direction of influence by shape and the DoN by color $(\diamond)$. Subsequently, to analyze the probability ranges of expenses, the organizers activate the distribution visualizations. Related cells then show the heatmaps of the distribution for the expenses. Upon analyzing the distribution, they realize that the value of the balance as shown in the spreadsheet is not very likely, and the computed meanbased on correct uncertainty propagation-is negative.

To evaluate how changes in the likelihoods for the expenses affect the balance, they enter the what-if analysis mode and change the DON to three to view the changes in all influencing cells. Their goal is to compare the scenario of the conference being held on site with the alternative of a virtual event.

To reflect the expected budget changes in the case of a virtual conference, the organizers start by reducing the expenses of venue and catering to almost zero with a high likelihood (see Figure 4, where the value of Catering is reduced to zero). This new value causes changes in the distributions of Catering, Total Expenses and Balance. They inspect the new distribution in the lower stack of the heatmap present in each of these cells and keep an eye on the side panel, as shown in Figure 4(C). To reflect the increased technical support needed for running the event virtually, they increase the corresponding cost. Since no local advertising is necessary and venue/catering expenses are reduced, conference sponsors will also pay less. Based on this information, the organizers obtain a more reliable (i.e., narrower) range of the balance. Additionally, registration costs per person are also to be reduced. After decreasing sponsorship amount and registration cost, the organizers try to increase the number of participants to make sure that the balance lies within a positive range, while being insensitive to minor fluctuations. Once the organizers are satisfied with their adapted planning, they decide to keep the updated values and save the changes in a separate file. The in-cell visualizations are preserved in the new file. They may choose to share this file with their colleagues, who can locally view the encodings even in the absence of the Fuzzy Spreadsheet add-on.

Once familiar with these encodings, such as the relationship encodings for the influencing cells, it becomes easy to grasp the underlying computational graph. The impact encoding helps to identify the extent and nature of the influence a cell has on the reference cell. The changes made during the what-if analysis present in the form of change indicators provide an overview of what has changed and by how much. The stacked heatmap encodings in changed cells indicate how a value has become more certain. After sharing the findings, the organizers continue the analysis of the onsite conference scenario in the original file. In this case, the likelihood of expenses, such as Venue and Catering, can be increased, and the deviation in the amount of sponsorship can be decreased. This changes the probability distribution of the balance, which in turn makes the balance amount more certain.

This usage scenraio highlights the benefits of using Fuzzy Spreadsheet: hypothetical scenarios can be analyzed based on immediate visual and numerical feedback. This is especially important in the case of high-stakes investments, where changes are crucial to ensure a positive balance. The what-if analysis helps to determine sensitive values and to obtain a stable range of values for the final outcome without limiting the number of possible scenarios that can be investigated. Thus, Fuzzy Spreadsheet can improve the outcome of the decision-making process. 


\section{Evaluation}

The goal of the user study was to compare the performance of a standard spreadsheet tool with that of the Fuzzy Spreadsheet approach for the six analysis tasks summarized in Table 2 We chose Microsoft Excel as a representative spreadsheet tool, assuming that tools such as Google Sheets and Apache OpenOffice Calc would perform similarly. As Fuzzy Spreadsheet is built for casual users and is not meant to compete with specialized tools for expert analysis, such as Oracle Crystal Ball and @Risk, we did not choose them for the user study. Our main objective is to keep the familiar spreadsheet layout and to add uncertainty exploration. Therefore, we also dropped Guesstimate as a comparison tool, as it lacks spreadsheet features and focuses on the uncertainty analysis only. To avoid a learning effect, we used a between-subject design for comparing the two conditions (i.e., Excel with and without our extension). We conducted three pilot studies to test the functionality of the Fuzzy Spreadsheet, to fine-tune the levels of difficulty of the tasks, and to estimate the time needed for each participant. We incorporated the feedback regarding functionality and usability from the pilot into our prototype. To reduce the time per subject, we decided to reduce the number of questions to two per task, with additional two questions for the more high-level task T5 A dataset was chosen that could easily be understood by all participants. The context of the dataset was conference planning, as for the dataset described in Section 7 It contained three columns of forecast values with their corresponding likelihood and standard deviation values and fewer parameters than described in Section 7 We conducted the main study remotely with 14 participants (P1-P14; Gender: $\mathrm{m}=10, \mathrm{f}=4$; Age: $M=28.21, S D=$ 4.32 ), seven of which were assigned to Excel and seven to the Fuzzy Spreadsheet prototype. Two instructors were present throughout-one moderator guiding the participant through the experiment and a second for taking notes. We also like to point out that these studies were performed with some slightly different choices for the visual encoding. The initial design of the change indicators consisted of red and green glyphs that-despite their different shapes-could be hard to distinguish for people with color vision deficiency, which is why we changed them for the current version. Additionally, we fixed a minor error in the color schemes of the heatmaps.

We used a mixed-method approach to compare how both tools perform at each task in terms of three quantitative measures: (1) the ratio of correct answers in percent; (2) the response time in seconds, and (3) the mental effort required [31], measured on a seven-point Likert scale ranging from one (very low mental effort) to seven (very high mental effort). In addition, we assessed the System Usability Scale (SUS) score based on ten questions developed by Brooke et al. [20, p. 189ff]. Furthermore, we analyzed the thinking-aloud protocol, the feedback from questionnaires, and the follow-up interviews.

To assess the usability of Fuzzy Spreadsheet, we formulated four hypotheses, as listed in Table 3 H1 investigated the differences between a traditional spreadsheet in Excel and a Fuzzy Spreadsheet with regard to answer correctness. We expected Fuzzy Spreadsheet-with its in-cell visualiza- tions and the active legend-to show a higher answer correctness than Excel $\left(\mathrm{H} 1_{\mathrm{a}}\right)$. $\mathrm{H} 2$ evaluated the influence of our prototype on the response time. We presumed that, with the additional support provided by Fuzzy Spreadsheet, a faster response time can be achieved $\left(\mathrm{H} 2_{\mathrm{a}}\right)$. With $\mathrm{H} 3$, we tested whether Fuzzy Spreadsheet or traditional Excel requires a higher mental effort. Given that our approach uniquely combines visual support and calculation of changes (whatif analyses), we hypothesized that the mental effort required is lower with Fuzzy Spreadsheet than with Excel $\left(\mathrm{H} 3_{\mathrm{a}}\right)$. With H4, we examined statistical differences between the two systems with regard to the SUS score. Based on these hypotheses, we expected Fuzzy Spreadsheet to achieve a higher SUS score than Excel $\left(\mathrm{H}_{\mathrm{a}}\right)$.

\subsection{Procedure}

To make the results comparable, we followed a similar procedure for both conditions, as illustrated in Figure 5. On enrollment in the study, each participant received an explanation of the study goals and the procedures, and was given access to the dataset loaded into the spreadsheet. This introduction also incorporated the nomenclature and the formulas used. For the Fuzzy Spreadsheet prototype, we provided an additional explanation of the visual encodings in the side panel and the in-cell visualizations for relationship, impact, likelihood, and distribution. For the Fuzzy Spreadsheet participants, we installed our extension on the participants' computers and verified functionality by means of a test file. After successful set-up and introduction, we asked the participants to perform the six analysis tasks. To avoid a selection bias, tasks were assigned in random order. In the course of the study, we recorded (i) externalized knowledge in a thinking-aloud protocol and (ii) the mental effort required for each task. After completion of the tasks, we gathered data for the SUS score and demographics in a survey, and performed semi-structured interviews to gain further insights into the usage of uncertain calculations. We closed each session with a debriefing.

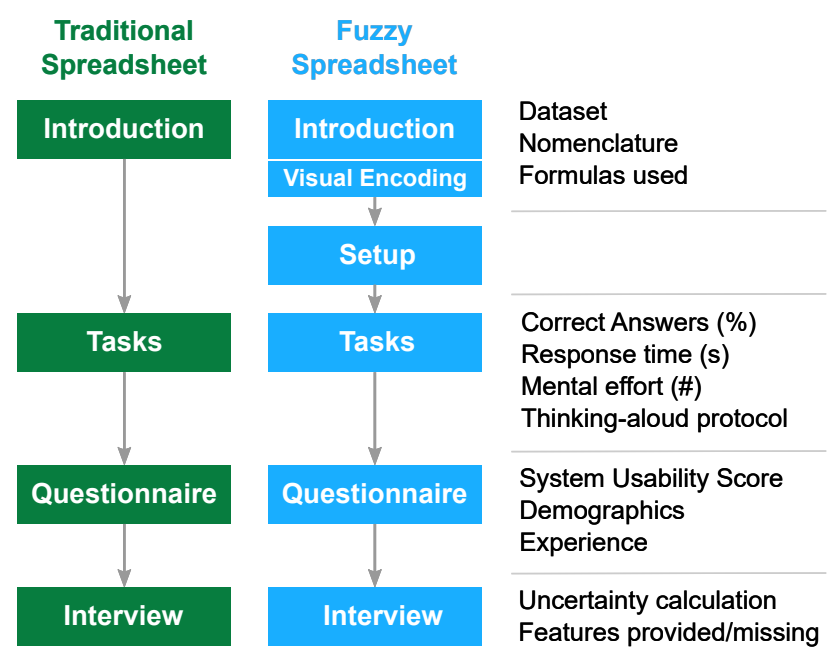

Fig. 5. Study procedure for both systems, Excel and Fuzzy Spreadsheet, starting with an introduction followed by task completion, a questionnaire, and an interview. 
TABLE 3

Overview of the hypotheses tested in our user study, with $\checkmark$ and $\boldsymbol{X}$ indicated accepted and rejected hypotheses, respectively.

\begin{tabular}{llll}
\hline$\#$ & Hypothesis & Accept/Reject \\
\hline $\mathbf{H} \mathbf{1}_{\mathbf{0}}$ & There is no statistically significant difference regarding answer correctness between the Excel system and Fuzzy Spreadsheet. & $\boldsymbol{x}$ \\
$\mathbf{H} \mathbf{1}_{\mathbf{a}}$ & Fuzzy Spreadsheet shows a statistically significantly higher answer correctness than the traditional spreadsheet. & $\checkmark$ \\
\hline $\mathbf{H} \mathbf{2}_{\mathbf{0}}$ & There is no statistically significant difference in response time between the Excel system and Fuzzy Spreadsheet. & $\boldsymbol{x}$ \\
$\mathbf{H} \mathbf{2}_{\mathbf{a}}$ & Fuzzy Spreadsheet shows a statistically significantly lower response time than the traditional spreadsheet. & $\checkmark$ \\
\hline $\mathbf{H} \mathbf{3}_{0}$ & There is no statistically significant difference in the mental effort required between the Excel system and Fuzzy Spreadsheet. & $\boldsymbol{x}$ \\
$\mathbf{H} \mathbf{3}_{\mathbf{a}}$ & Fuzzy Spreadsheet requires a statistically significantly lower mental effort than the traditional spreadsheet. & $\checkmark$ \\
\hline $\mathbf{H} \mathbf{4}_{\mathbf{0}}$ & There is no statistically significant difference in the SUS score between the Excel system and Fuzzy Spreadsheet. \\
$\mathbf{H} \mathbf{4}_{\mathbf{a}}$ & Fuzzy Spreadsheet shows a statistically significantly higher SUS score than the traditional spreadsheet. & $\boldsymbol{x}$ \\
\hline
\end{tabular}

\subsection{Setup}

As both conditions were based on Excel, we created one worksheet per question in a single file. For a fair comparison, we developed a Visual Basic for Applications (VBA) script which recorded the answers, checked for correctness, and recorded the time until answer submission. Responsetime tracking started with the activation of a sheet and ended when a participant submitted an answer. In addition, the script stored the Likert scale value for the mental effort required. Since this was a remote laboratory experiment, we performed and recorded each session using the video conference platform Zoom [49]. The moderator shared the screen to introduce the prototype features to the participants. During the study, we asked the participants to share their screens so we could observe their actions. If the installation of the extension did not work properly due to incompatible system requirements, participants were asked to connect to the moderator's computer via TeamViewer [42].We employed the online and open-source survey tool LimeSurvey [24] for scoring the ten items from the System Usability Scale, for capturing the demographics, and for checking the participants' levels of experience with spreadsheet tools, data visualization, and data science.

\subsection{Study Results}

To assess differences between the two conditions, we used a $\chi^{2}$ independence test for our dichotomous variable answer correctness. Further, to analyze differences in the response time, in the mental effort captured with a seven-point Likert scale and in the SUS scores, we applied a Student's $t$-test. The results at task level were examined using a Mann-Whitney $U$ test for answer correctness and a oneway Analysis of Variance (ANOVA) for both response time and mental effort required. Further, we analyzed behavioral observations from the user studies and the thinking-aloud protocol.

\subsubsection{Tool Comparison}

First, we analyzed the significance of differences between the two tools in terms of answer correctness. We ascertained that answer correctness with Fuzzy Spreadsheet was statistically significantly higher than that of the standard Excel condition $\left(\chi^{2}(1)=21.448, p=.000\right)$, based on which we reject our null-hypothesis $H 1_{0}$ and accept $H 1_{a}$.
Based on our analysis of response times, we can also reject $\mathrm{H} 2_{0}$. With Fuzzy Spreadsheet, the mean response time was $102.21 \mathrm{~s}(S D=57.90 \mathrm{~s})$, which is significantly shorter than the response time for the Excel tool $(M=170.60 \mathrm{~s}$, $S D=142.54 \mathrm{~s}, t(184)=-4.286, p=.000$; accept $\left.\mathrm{H} 2_{\mathrm{a}}\right)$.

Subsequently, we took a closer look at the subjective measure of mental effort required, and investigated the difference between the two tools. There was a significant difference in mental effort required for the Fuzzy Spreadsheet $(M=2.94, S D=1.23)$ and the traditional spreadsheet $(M=3.44, S D=1.77)$ conditions $(t(188)=-2.260$, $p=.025)$, leading us to reject $\mathrm{H} 3_{0}$ and accept $\mathrm{H} 3_{\mathrm{a}}$. These results coincide with our observation that Fuzzy Spreadsheet was also used as an affirmative support tool. Participants first sought to calculate the results in their heads before confirming them with the extension. Thus, the mental effort was perceived to be lower with Fuzzy Spreadsheet than with Excel. In general, participants in both groups tried to rely on prior knowledge to answer questions about the financial model without recourse to the extension. Further, participants from the Excel control group made supplementary calculations, either directly in Excel or with external tools, such as calculators or Wolfram Alpha [47|. Participants using Fuzzy Spreadsheet scored statistically significantly higher on the System Usability Scale $(M=78.57$, $S D=15.27)$ than participants using a standard spreadsheet $(M=48.57, S D=15.93, t(12)=3.597, p=.004)$. Hence, we can also reject $\mathrm{H} 4_{0}$ and accept $\mathrm{H} 4_{\mathrm{a}}$. According to the SUS scores, Fuzzy Spreadsheet was classified as a good and the traditional Excel as an awful tool for the given tasks.

In summary, Fuzzy Spreadsheet had a higher response accuracy, shorter response time, required an overall lower mental effort, and was rated with a higher SUS score than Excel.

\subsubsection{Task Comparison}

We noticed that some tasks were performed more easily with one tool than with the other one. Thus, we decided to assess the applicability of our spreadsheet augmentation in a fine-grained analysis. Based on our quantitative measures, we determined differences between the six tasks.

As indicated in Figure 6. Fuzzy Spreadsheet outperformed the traditional spreadsheet in all tasks. Especially the low response accuracy below $50 \%$ for $\mathbf{T 2}$ and T4 in the Excel group is noticeable. On closer examination, we 
found that participants took statistically significantly longer $(M=351.47 \mathrm{~s}, S D=196.84 \mathrm{~s})$ for $\mathrm{T3}$ with the traditional spreadsheet than for the other tasks $(F(25,13,597)=$ $18,540, p=.000)$. This is consistent with our observations and the thinking-aloud protocol. Participants using Excel without Fuzzy Spreadsheet stated that an impact in percent "is difficult to compute, especially in the case of higher DoNs" (P6-P10). We also determined-by means of a posthoc Tukey HSD test-that Assess Impact (T3) was not only the task that showed the longest response time, but also the task which required the highest mental effort for Excel users $(M=5.46, S D=1.61)$. All participants in the control group used auxiliary calculations to answer questions related to this task, while participants from the Fuzzy Spreadsheet condition used the side panel as an additional confirmatory tool. In contrast, Lookup Value (T1) tasks were perceived as the least demanding by Excel users.

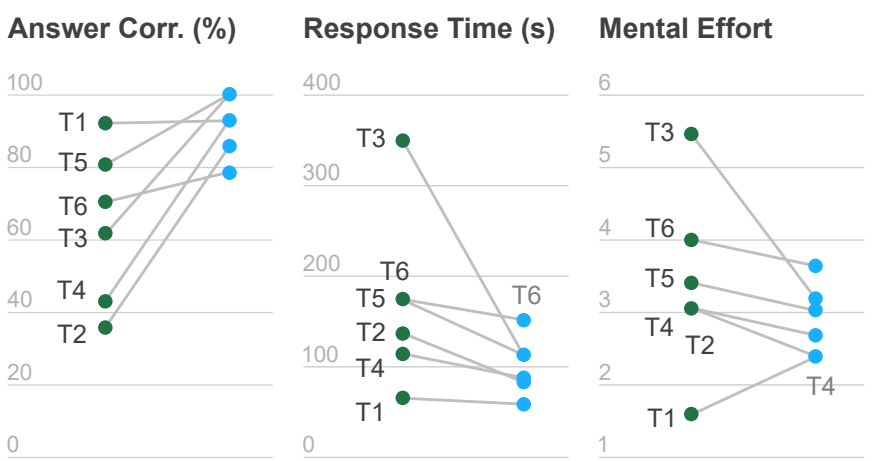

Fig. 6. Differences in answer correctness, response time, and mental effort between Excel and Fuzzy Spreadsheet for each analysis task T1 T6].

Overall, Fuzzy Spreadsheet led to higher response accuracies, shorter response times, and required lower mental effort (except for T1.

\subsubsection{Behavioral patterns}

To further describe differences between the Excel and the Fuzzy Spreadsheet condition, we analyzed the thinkingaloud protocols and the interviews. We discuss behavioral patterns that we observed while participants performed the tasks.

Observations of the control group showed behavioral patterns with the traditional spreadsheet, which we had anticipated and addressed with Fuzzy Spreadsheet. For instance, we observed that all participants from the Fuzzy Spreadsheet condition activated the relationship and neighborhood encoding for each task by default. In contrast, participants who used the Excel tool had "difficulties in incorporating the probability/likelihood and further determining the impact" (translated from German) (T3). In particular, P10 mentioned that it was unclear "how one can determine the impact of a neighbor by taking both likelihood and standard deviation into account" (translated from German). This can be traced back to a generally reduced understanding of probability calculation $(\mathrm{P} 8, \mathrm{P} 9)[15]$ or understanding problems of uncertainty propagation ( $\overrightarrow{\mathrm{P} 1}$, P4, P5, P6, P9, P11) [48]. The encoding selected to indicate influencing and influenced by cells-glyphs with a gray value for the DoN-was received particularly well and considered to be very helpful. However, some participants (P1, P2, P14) were confused by the placement of the glyphs on the far left edges of the cells. If only the relationship glyphs were displayed and no other visual support was enabled, they tended to associate the glyphs with the cell to the left rather than the selected cell itself. Interestingly, observations from the Excel control group revealed that two participants (P8 and P11) introduced similar highlighting for related cells themselves. They used different shades for the DoN to facilitate fast detection of coherent cells. P11 from the Excel group even colored reference and selected cells in a consistent color scheme across all task, which is reminiscent of our highlighting approach. Participants who did not mark important cells, needed longer to complete the task. These observations support our design choice of introducing distinct colors for the different cell types.

We further gained insight into how Excel users performed Formulate Cause and Effect tasks (T5). In the course of the analysis, two participants (P8 and P10) realized that they forgot to write down the initial values. Participants typically took notes next to the financial dashboard to reliably track and compare value changes (P8, P9, P10) and to summarize their insights (P8, P9). This way, they were able to compare an initial value with a newly calculated one. Furthermore, participants mostly entered initial values in hard-coded form (P6, P7, P8), regardless of the level of familiarity with Excel. This static note-taking approach made it difficult for participants to compare and track changes, particularly for tasks that included secondary calculations or even an increased degree of neighborhood.

\subsection{Summary of Findings}

Fuzzy Spreadsheet makes it easier to understand the information about the underlying relationships between cells and to explore the propagation of uncertainty. Most importantly, the user study indicates that the correctness of answers can be significantly influenced by computational and visual support. Necessary functionalities that we anticipated in our Fuzzy Spreadsheet approach and that are available only to a limited extent in traditional spreadsheets were well received. In particular, the interviewees considered the glyph encodings applied to the impact (P1, P2, P4, P5), directed relationships with neighborhood information (P2, P4), and the what-if analyses for assessing variations (P3, P5) as the key features of Fuzzy Spreadsheet. In summary, learning from our results, we recommend holding on to the familiar tools of the spreadsheet and augmenting these established designs judiciously and with great care to support encodings of visual.

\section{Discussion}

In this section, we discuss conceptual limitations of the overall approach and technical limitations of the prototype implementation.

Arithmetic Operations: For this work, we focused on making the visual encoding and the uncertainty propagation easy to understand. To this end, we relied on spreadsheets that include basic mathematical operations (addition, 
subtraction, multiplication and average). We plan to extend the functionality of Fuzzy Spreadsheet by also incorporating more complex arithmetic operations. This is not straightforward because it requires a more sophisticated sampling and parsing process for calculating the distributions.

Uncertainty Definition: As described in Section 4.1. the extension assigns specific meaning to the cells to categorize them as uncertain cells. It supports only two types of distributions with which fuzzy cells can be initializedNormal and Bernoulli distributions (reflected by the mean, standard deviation, and likelihood columns). To cover a wider range of scenarios, users need to flexibly specify general distributions for cells, as supported by other tools, such as Oracle Crystal Ball, @RISK, and Guesstimate.

Uncertainty Analysis over Time: By using standard spreadsheet functionality, users can create predictions based on time-series data. Fuzzy Spreadsheet does not yet provide a way to display and explore the uncertainties over time. In the future, we plan to incorporate temporal aspects into our uncertainty visualizations.

Performance Limitations: The performance of the extension depends on the amount of data, the sampling approach, and the platform used. For a small data set $(\sim 30$ rows and $\sim 20$ columns), it shows a significantly better performance in the Excel desktop version than in the Excel online version. The desktop version needs approximately 1.2 seconds on a standard laptop computer for parsing, preprocessing, and displaying all data from the budget calculation example shown in Figure 4 . The same operations take approximately ten seconds with the online version. The reason for the performance difference is the online version's auto-save mode, which cannot be disabled and constantly interferes with the construction of the visualizations. If the size of the spreadsheet or the number of samples generated during the uncertainty propagation increases, the performance drops considerably in both the Excel desktop and the online version. To address this problem, we plan to make use of a remote service for parsing the data and carrying out the more demanding operations such as sampling.

\section{Conclusion}

Tracing uncertainty in spreadsheets is a challenging task that arises in many application contexts. In this paper, we have presented Fuzzy Spreadsheet, an augmentation approach that adds in-cell visualizations to communicate sensitivity and robustness information while staying as close to the familiar spreadsheet layout as possible. Fuzzy Spreadsheet allows users to track the propagation of uncertainty information through a spreadsheet and to compare alternative scenarios as part of what-if analyses.

To evaluate the efficacy of our solution, we performed a small-scale user study that compared the Fuzzy Spreadsheet approach with traditional spreadsheets in terms of answer correctness, response time, mental effort, and usability. In summary, the results indicate that Fuzzy Spreadsheet outperforms traditional spreadsheets and empowers users to carry out tasks related to tracking and exploring uncertainty information more effectively.

\section{RefEREnCES}

[1] R. Amar and J. Stasko. A Knowledge Task-Based Framework for Design and Evaluation of Information Visualizations. In IEEE Symposium on Information Visualization, pp. 143-150, Oct. 2004. Citation Key Alias: amar_knowledge_2004. doi: 10.1109/INFVIS .2004 .10

[2] J. Bilcke, P. Beutels, M. Brisson, and M. Jit. Accounting for Methodological, Structural, and Parameter Uncertainty in DecisionAnalytic Models: A Practical Guide. Medical Decision Making, 31(4):675-692, 2011. doi: 10.1177/0272989X11409240

[3] G.-P. Bonneau, H.-C. Hege, C. R. Johnson, M. M. Oliveira, K. Potter, P. Rheingans, and T. Schultz. Overview and State-of-the-Art of Uncertainty Visualization. In C. D. Hansen, M. Chen, C. R. Johnson, A. E. Kaufman, and H. Hagen, eds., Scientific Visualization, pp. 3-27. Springer, London, 2014. Series Title: Mathematics and Visualization.

[4] R. Borgo, J. Kehrer, D. H. S. Chung, E. Maguire, R. S. Laramee, H. Hauser, M. Ward, and M. Chen. Glyph-based Visualization: Foundations, Design Guidelines, Techniques and Applications. Eurographics 2013 - State of the Art Reports, 2012. doi: 10.2312/ CONF/EG2013/STARS/039-063

[5] M. Bostock, V. Ogievetsky, and J. Heer. D3: Data-Driven Documents. IEEE Transactions on Visualization and Computer Graphics (InfoVis '11), 17(12):2301-2309, 2011. doi: 10.1109/TVCG.2011.185

[6] M. Brehmer and T. Munzner. A Multi-Level Typology of Abstract Visualization Tasks. IEEE Trans. Visual. Comput. Graphics, 19(12):2376-2385, Dec. 2013. doi: 10.1109/TVCG.2013.124

[7] H. Chen, S. Zhang, W. Chen, H. Mei, J. Zhang, A. Mercer, R. Liang, and H. Qu. Uncertainty-Aware Multidimensional Ensemble Data Visualization and Exploration. IEEE Transactions on Visualization and Computer Graphics, 21(9):1072-1086, 2015. doi: 10.1109/TVCG. 2015.2410278

[8] R. A. Choudhury, W. F. Mahaffee, N. McRoberts, and W. D. Gubler. Modeling Uncertainty in Grapevine Powdery Mildew Epidemiology Using Fuzzy Logic. BioRxiv, 264622, 2018. doi: 10. $1101 / 264622$

[9] J. de Jong. math.js - an extensive math library for JavaScript and Node.js. https://mathjs.org/ 2019.

[10] D. Feng, L. Kwock, Y. Lee, and R. M. Taylor. Matching Visual Saliency to Confidence in Plots of Uncertain Data. IEEE Transactions on Visualization and Computer Graphics, 16(6):980-989, 2010. doi: 10.1109/TVCG.2010.176

[11] M. Fernandes, L. Walls, S. Munson, J. Hullman, and M. Kay. Uncertainty Displays Using Quantile Dotplots or CDFs Improve Transit Decision-Making. In Proceedings of the ACM SIGCHI Conference on Human Factors in Computing Systems (CHI '18), pp. 1-12. ACM Press, 2018. doi: 10.1145/3173574.3173718

[12] N. French and L. Gabrielli. Discounted cash flow: accounting for uncertainty. Journal of Property Investment E Finance, 23(1):75-89, 2005. doi: $10.1108 / 14635780510575102$

[13] K. Furmanova, S. Gratzl, H. Stitz, T. Zichner, M. Jaresova, M. Ennemoser, A. Lex, and M. Streit. Taggle: Scalable Visualization of Tabular Data through Aggregation. Information Visualization, 2019. doi: $10.1177 / 1473871619878085$

[14] A. Gogolou, T. Tsandilas, T. Palpanas, and A. Bezerianos. Comparing Similarity Perception in Time Series Visualizations. IEEE Transactions on Visualization and Computer Graphics (InfoVis '18), 25(1):523-533, 2019. doi: 10.1109/TVCG.2018.2865077

[15] D. G. Goldstein and D. Rothschild. Lay understanding of probability distributions. Judgment and Decision Making, 9(1):14, 2014.

[16] Google LLC. Google Sheets. https://www.google.com/sheets/ about/

[17] Guesstimate Inc. Guesstimate. https://www.getguesstimate. com/. 2019.

[18] J. L. Hintze and R. D. Nelson. Violin Plots: A Box Plot-Density Trace Synergism. The American Statistician, 52(2):181-184, 1998. doi: 10.1080/00031305.1998.10480559

[19] C. Holzhüter, A. Lex, D. Schmalstieg, H.-J. Schulz, H. Schumann, and M. Streit. Visualizing Uncertainty in Biological Expression Data. In Visualization and Data Analysis, p. 82940O. International Society for Optics and Photonics, 2012. doi: 10.1117/12.908516

[20] P. W. Jordan, ed. Usability evaluation in industry. CRC Press, 1996.

[21] M. Knauff and A. G. Wolf. Complex cognition: the science of human reasoning, problem-solving, and decision-making. Cognitive Processing, 11(2):99-102, 2010. doi: 10.1007/s10339-010-0362-z 
[22] A. Kohlhase. Human-Spreadsheet Interaction. In P. Kotzé, G. Marsden, G. Lindgaard, J. Wesson, and M. Winckler, eds., Human-Computer Interaction - INTERACT 2013, Lecture Notes in Computer Science, pp. 571-578. Springer, 2013. doi: 10.1007/978-3 $-642-40498-6 \_47$

[23] A. Lex, C. Partl, D. Kalkofen, M. Streit, S. Gratzl, A. M. Wassermann, D. Schmalstieg, and H. Pfister. Entourage: Visualizing Relationships between Biological Pathways using Contextual Subsets. IEEE Transactions on Visualization and Computer Graphics (InfoVis '13), 19(12):2536-2545, 2013. doi: 10.1109/TVCG.2013.154

[24] LimeSurvey $\mathrm{GmbH}$. Professionelle Online-Umfragen mit LimeSurvey. https://www.limesurvey.org/. 2020.

[25] C. Lundström, P. Ljung, A. Persson, and A. Ynnerman. Uncertainty Visualization in Medical Volume Rendering Using Probabilistic Animation. IEEE Transactions on Visualization and Computer Graphics (Vis '07), 13(6):1648-1655, 2007. doi: 10.1109/TVCG.2007. 70518

[26] J. Menick. sampling - JavaScript for sampling from a handful of discrete probability distributions. https://github.com/ jacobmenick/sampling 2016.

[27] Microsoft Corporation. Microsoft Excel. https://www.microsoft. com/en-us/microsoft-365/excel/

[28] Microsoft Corporation. Understanding the Office JavaScript API. https://docs.microsoft.com/en-us/office/dev/add-ins/ develop/understanding-the-javascript-api-for-office/. 2020.

[29] C. Nobre, M. Streit, and A. Lex. Juniper: A Tree+Table Approach to Multivariate Graph Visualization. Transaction on Visualization and Computer Graphics (InfoVis '18), 25(1):544 - 554, 2019. doi: 10. 1109/TVCG.2018.2865149

[30] Oracle Corporation. Oracle Crystal Ball. https://www.oracle. com/applications/crystalball/. 2020.

[31] F. G. W. C. Paas, J. J. G. van Merriënboer, and J. J. Adam. Measurement of Cognitive Load in Instructional Research. Perceptual and Motor Skills, 79(1):419-430, 1994. doi: 10.2466/pms.1994.79.1.419

[32] Palisade. @RISK. https://www.palisade.com/risk/. 2020.

[33] A. T. Pang, C. M. Wittenbrink, and S. K. Lodh. Approaches to Uncertainty Visualization. The Visual Computer, 13(8):370-390, 1997.

[34] A. Parush, A. Hod, and A. Shtub. Impact of visualization type and contextual factors on performance with enterprise resource planning systems. Computers $\mathcal{E}$ Industrial Engineering, 52(1):133142, 2007. doi: 10.1016/j.cie.2006.11.002

[35] C. V. Phillips and L. M. LaPole. Quantifying errors without random sampling. BMC Medical Research Methodology, 3(1):9, 2003. doi: 10.1186/1471-2288-3-9

[36] T. Pitcher. Beverton and Holt Equations: Spreadsheet functions and Uncertainty, Mar. 1999.

[37] K. Potter, P. Rosen, and C. R. Johnson. From Quantification to Visualization: A Taxonomy of Uncertainty Visualization Approaches. In Uncertainty Quantification in Scientific Computing, pp. 226-249. Springer, London, 2012.

[38] J. Sanyal, Song Zhang, G. Bhattacharya, P. Amburn, and R. Moorhead. A User Study to Compare Four Uncertainty Visualization Methods for 1D and 2D Datasets. IEEE Transactions on Visualization and Computer Graphics, 15(6):1209-1218, 2009. doi: 10.1109/TVCG. 2009.114

[39] J. Sanyal, Song Zhang, J. Dyer, A. Mercer, P. Amburn, and R. J. Moorhead. Noodles: A Tool for Visualization of Numerical Weather Model Ensemble Uncertainty. IEEE Transactions on Visualization and Computer Graphics, 16(6):1421-1430, 2010. doi: 10. 1109/TVCG.2010.181

[40] D. Spiegelhalter, M. Pearson, and I. Short. Visualizing Uncertainty About the Future. Science, 333(6048):1393-1400, 2011. doi: 10.1126/ science. 1191181

[41] A. Streit, Binh Pham, and R. Brown. A Spreadsheet Approach to Facilitate Visualization of Uncertainty in Information. IEEE Transactions on Visualization and Computer Graphics, 14(1):61-72, 2008. doi: 10.1109/TVCG.2007.70426

[42] TeamViewer Germany GmbH. TeamViewer. https://www. teamviewer.com/. 2020.

[43] The Apache Software Foundation. Apache OpenOffice Calc. https: //www.openoffice.org/product/calc.html

[44] D. van der Laan, E. de Jonge, and J. Solcer. Effect of Displaying Uncertainty in Line and Bar Charts - Presentation and Interpretation: In Conference on Information Visualization Theory and Applications (IVAPP '15), pp. 225-232, 2015. doi: 10.5220/0005300702250232
[45] Z. Vosough, D. Kammer, M. Keck, and R. Groh. Visualizing uncertainty in flow diagrams: a case study in product costing. In Symposium on Visual Information Communication and Interaction (VINCI '17), pp. 1-8. ACM Press, 2017. doi: 10.1145/3105971. 3105972

[46] M. Williams and T. Norris. jStat - JavaScript statistical library. https://github.com/jstat/jstat 2020.

[47] Woltram Alpha LLC. WolframAlpha. https://www. wolframalpha.com/

[48] Y. Wu, G.-X. Yuan, and K.-L. Ma. Visualizing Flow of Uncertainty through Analytical Processes. IEEE Transactions on Visualization and Computer Graphics (InfoVis '12), 18(12):2526 -2535, 2012. doi: 10 .1109/TVCG.2012.285

[49] Zoom Video Communications, Inc. Zoom. https://zoom.us/ 2020. 